presentation attending the paediatric neurology departments in Ireland. A questionnaire and chart review was performed on all cases.

Results 16 cases were documented by paediatric neurologists from 2006- 2013 giving an incidence of 0.21/100,000. 13 occurred in females with a median age of 10 years. $75 \%$ presented predominantly with a hemichorea.

All had functional impairment with 11/16 having behavioural or mood change. 5 had carditis diagnosed by echo. 13 had serological evidence of previous GAS infection. All had a normal MRI brain.

$15 / 16$ received medication. valproate was the first choice in 11 cases, steroids in 2 and valproate in combination with steroids in 2.8 were treated with penicillin for $>11$ months.

Conclusion The incidence of Sydenhams chorea appears to be rising. It is a clinical diagnosis and important to recognise. In our cohort we used a combination of steroids and valproate which gave a good response. There was a trend towards longterm penicillin.

Acknowledgements Collaborators: David Webb (Crumlin), Tony Bouldin (Crumlin), Deidre Peake (Belfast), Sanjay Tirupathi (Belfast), Niamh Mc Sweeney (Cork).

\section{PS-250 WHAT ADULTS KNOW ABOUT CHILD DEVELOPMENT IN ALBERTA, CANADA: IMPLICATIONS FOR HEALTH SERVICES}

${ }^{1} \mathrm{~A}$ Pujadas Botey, ${ }^{2}$ WB Gibbard, ${ }^{2} \mathrm{~K}$ MacLellan, ${ }^{3} \mathrm{C}$ Bukutu, ${ }^{2} \mathrm{H}$ Bayrampour, ${ }^{2} \mathrm{~A}$ Vinturache, ${ }^{4}$ D Slater, ${ }^{5} \mathrm{R}$ Breitkreuz, ${ }^{5} \mathrm{~L}$ Sakaluk-Moody, ${ }^{6} \mathrm{~S}$ Lynch, ${ }^{7} \mathrm{~S}$ Tough. ${ }^{1}$ Alberta Centre for Child Family and Community Research, Calgary, Canada; ${ }^{2}$ Paediatrics, University of Calgary, Calgary, Canada; ${ }^{3}$ Child and Youth Data Laboratory, Alberta Centre for Child Family and Community Research, Edmonton, Canada; ${ }^{4}$ Physiology and Pharmacology, University of Calgary, Calgary, Canada; ${ }^{5}$ Human Ecology, University of Alberta, Edmonton, Canada; ${ }^{6}$ Community-University Partnership for the Study of Children Youth and Families, University of Alberta, Edmonton, Canada; ${ }^{7}$ Paediatrics and Community Health Sciences, University of Calgary and Alberta Centre for Child Family and Community Research, Calgary, Canada

\subsection{6/archdischild-2014-307384.549}

Background Adult knowledge of child development shapes their expectations and feedback to children as they learn and grow. Effective guidance of children by adults is influenced by their knowledge of child development.

Study questions/aims (1) What is adult Albertans' knowledge of developmental milestones from birth to 6-years in four developmental domains: physical, cognitive/language, social, and emotional? (2) What parenting supports do adults use regarding child development?

Methods 1,451 randomly selected adults in Alberta, both parents and non-parents, completed a telephone survey exploring knowledge about child development and parenting supports. Data was analysed using univariate and multivariate techniques $(\mathrm{p}<0.05)$.

Results $35 \%$ of adults identified when at least half of physical developmental milestones typically occur; 23\%, 16\%, and 11\% could do so for cognitive/language, emotional, and social development milestones respectively. Errors in reporting when milestones typically occur were primarily related to respondents thinking a milestone was achieved earlier. Knowledge has not changed since a similar survey in 2007. Gaps in child development knowledge did not differ substantively between parents/ non-parents, females/males or urban/rural residence. Respondents identified key parenting supports to be family members
(76\%), family doctors or paediatricians (61\%), and teachers (54\%). Common resources included books (68\%), television/ media (43\%), and the internet (36\%).

Conclusions There were meaningful gaps in adult knowledge of when children achieve developmental milestones. Evidence to date suggests that better child outcomes begin with accurate parent knowledge of child development. There is opportunity to help parents and care providers become more aware of these milestones through knowledge dissemination strategies.

\section{PS-251 DEVELOPMENTAL COORDINATION DISORDER IN CHILDREN WITH A HISTORY OF INFANTILE COLIC}

${ }^{1}$ I Milidou, ${ }^{1} \mathrm{MS}$ Jensen, ${ }^{2} \mathrm{C}$ Søndergaard, ${ }^{3} \mathrm{~J}$ Olesen, ${ }^{1} \mathrm{~TB}$ Henriksen. ${ }^{1}$ Department of Paediatrics, Aarhus University Hospital, Aarhus N, Denmark; ' ${ }^{2}$ epartment of Paediatrics, Herning Regional Hospital, Herning, Denmark; ${ }^{3}$ Institute of Public Health, Aarhus University, Aarhus, Denmark

\subsection{6/archdischild-2014-307384.550}

Background Infantile colic or excessive crying during the first months of life is common and causes concern among parents. Little is known about the health and development of children with a history of infantile colic. We evaluated whether these children have motor development impairments by the age of seven. Method Data on crying symptoms in infancy and parental Developmental Coordination Disorder Questionnaire '07 (DCDQ'07) were avaliable for 27,940 singletons from the Danish National Birth Cohort. We fitted a linear regression comparing DCDQ'07 totl scores of children with and without a history of infantile colic. We moreover compared the risk (odds) for probable Developmental Coordination Disorder (DCD), defined as a DCDQ'07 total score $<46$, for children with and without a history of infantile colic in a logistic regression model. All analyses were adjusted for a number of covariates.

Results Children with a history of infantile colic had slightly lower total DCDQ'07 scores (-0.5 [95\% confidence interval: $0.9 ;-0.03]$. The difference was larger among boys (-0.7 [-1.4; $0.1])$ than among girls $(-0.2[-0.7 ; 0.3])$. Children with a history of infantile colic had slightly higher risk for probable DCD (Odds ratio: 1.2 [1.0; 1.6]. This was more obvious among boys $(1.3[1.0 ; 1.7])$ than among girls $(1.0[0.6 ; 1.7])$. For both associations, the gender-specific estimates did not differ statistically.

Conclusion Boys with a history of infantile colic had a tendency to lower motor development scores. However, the associations were weak, and the clinical importance of these findings may be limitted.

\section{PS-252 INFANTILE COLIC, HYPERKINETIC DISORDER, AND EMOTIONAL AND BEHAVIOURAL PROBLEMS IN CHILDHOOD - THE DANISH NATIONAL BIRTHCOHORT}

${ }^{1}$ I Milidou, ${ }^{2} \mathrm{C}$ Søndergaard, ${ }^{1} \mathrm{MS}$ Jensen, ${ }^{3} \mathrm{Z}$ Liew, ${ }^{4} \mathrm{~J}$ Olesen, ${ }^{1} \mathrm{~TB}$ Henriksen. ${ }^{1}$ Department of Paediatrics, Aarhus University Hospital, Aarhus N, Denmark; ${ }^{2}$ Department of Paediatrics, Herning Regional Hospital, Herning, Denmark; ${ }^{3}$ Department of Epidemiology School of Public Health, University of California, Los Angeles, USA; ${ }^{4}$ Department of Epidemiology School of Public Health, Aarhus University, Aarhus, Denmark

\subsection{6/archdischild-2014-307384.551}

Background Infantile colic is considered a transient problem, but children with infantile colic may have persistent behavioural problems. We studied the association between history of infantile colic, and attention deficit, hyperactivity, emotional and behavioural problems. 
Method Data on crying symptoms in infacy and Strengths and Difficulties Questionnaire (SDQ) scores at the age of 7 were available for 39,000 singletons from the Danish National Birth Cohort (DNBC). SDQ scores above the 90th percentile of community samples were characterised as abnormal. We compared the odds for abnormal SDQ scores of children with and without infantile colic using logistic regression.

Complete follow-up with hospital admissions and discharge diagnoses from the National Patients' Register were available for 63,773 children from the DNBC with data on crying symptoms. Hazard ratios (HR) for diagnosis of hyperkinetic disorder (HKD, ICD-10 F90.0-F90.9) were estimated using Cox regression.

Results Children with a history of infantile colic had an increased risk of abnormal scores in all SDQ difficulties subscales (OR [95\% confidence intervals]: emotional symptoms: 1.6 [1.41.8], conduct problems: 1.5 [1.3-1.7], attention deficit/hyperactivity: 1.5 [1.3-1.8], relation with peers:1.4 [1.2-1.6]), as well as in the Total Difficulties score (1.6 [1.4-1.9].

We moreover observed an increased risk of HKD diagnosis (HR: 1.4 [1.1-1.9] among children with a history of infantile colic. Results were similar after adjustment for gestational age, birth weight, several intrauterine exposures, and maternal SDQ symptoms.

Conclusion Children with a history of infantile colic had higher risk of emotional, behavioural, attention deficit/hyperactivity problems, and for being diagnosed with HKD.

\section{PS-253 AUTISTIC FEATURES, PERSONALITY AND RISK TAKING OF A VERY PRETERM AND/OR VERY LOW BIRTH WEIGHT COMMUNITY SAMPLE OF ADULTS}

${ }^{1} \mathrm{~S}$ Eryigit-Madzwamuse, 'D Wolke, ${ }^{1} \mathrm{~N}$ Baumann, ${ }^{2} \mathrm{P}$ Bartmann. ' ${ }^{1}$ sychology, University of Warwick, Coventry, UK; ${ }^{2}$ Neonatology, University Hospital Bonn, Bonn, Germany

\subsection{6/archdischild-2014-307384.552}

Background and aims Previously low risk-taking was reported in very preterm (VP: gestational age at-birth $<32$ weeks) and/or very low birth-weight (VLBW: birth-weight $<1500$ grams; subsequently $\mathrm{VP} / \mathrm{VLBW}$ ) samples. Unknown is whether there are other specific personality characteristics associated with VP/ VLBW adults. The aim of this study was to examine autistic features, personality characteristics and risk-taking attitudes of $\mathrm{VP} /$ VLBW in comparison to full-term controls at 26 years of age.

Methods The Bavarian Longitudinal Study is a geographically defined prospective whole population sample of neonatal at-risk children born in Germany. The sample comprised of $200 \mathrm{VP} /$ VLBW adults and 197 controls. The test battery included the Broad Autism Phenotype Questionnaire (autistic features), the Big-5 Inventory (personality), and the Arnett Inventory of Sensation Seeking (risk-taking).

Results Compared to the control sample, VP/VLBW adults scored significantly higher in autism, introversion and neuroticism but not in conscientiousness and openness scales. They also reported decreased risk-taking. Profile analysis showed introversion, decreased risk-taking, autism and neuroticism as unique features of VP/VLBW compared to controls $\left(\mathrm{F}_{\text {within-group }}=0.70\right.$, $\left.n s ; \mathrm{F}_{\text {between-group }}=49.56, \mathrm{p}<0.001\right)$. These characteristics also loaded into a single profile factor $\left(\chi^{2}=7.99\right.$, $\mathrm{df}=4, n s$; $\mathrm{CFI}=$ $0.984)$. VP/VLBW birth significantly predicted the profile factor $(\beta=0.33, \mathrm{p}<0.001)$ and explained $11 \%$ of its variance.

Conclusions VP/VLBW birth poses an important risk for a global withdrawn personality, as indicated by being less socially engaged (introversion), low in taking risks, poor in communication (autistic features) and easily worried (neuroticism). This profile might help to explain the social difficulties VP/VLBW individuals experience in adult roles, such as in peer/partner relationships and career.

\section{PS-253a EXPRESSION OF CIRCULATING EHSC70 AND EHSP70 IN UMBILICAL ARTERY IS RELATED TO THE DEVELOPMENTAL QUOTIENT IN PRETERM-BORN CHILDREN}

${ }^{1}$ A Suryawan, ${ }^{1} \mathrm{MB}$ Narendra, ${ }^{1} \mathrm{MS}$ Subijanto, ${ }^{2} \mathrm{~S}$ Taat Putra. ${ }^{1}$ Pediatrics, Medical School of Airlangga University, Surabaya, Indonesia; ${ }^{2}$ Pathology Anatomy, Medical School of Airlangga University, Surabaya, Indonesia

\subsection{6/archdischild-2014-307384.553}

Background and aims Recent studies revealed that intracellular chaperones, heat shock protein $70 \mathrm{kD}$ family (constitutive Hsc70 and inducible Hsp70), may have specific role in brain development when they released into extracellular compartment (eHsc70 and eHsp70) since neurons and glial cells can take them up from blood circulation. The assessment of atypical brain development in high risk children require more precise quantitative developmental quotient (DQ) measures than qualitative categorical outcomes. The aim of this study was to determine the correlation between the expression of circulating eHsc70 and eHsp70 and the DQ in children who were born preterm.

Methods A total of 21 eligible preterm infants were studied prospectively. The expression of eHsc70, eHsp70 and eHsc + p70 (eHsc70+eHsp70) was determined on the basis of blood samples taken from umbilical artery at birth. The DQ was assessed using The Cognitive Adaptive Test/Clinical Linguistic and Auditory Milestone Scale (CAT/CLAMS) at the corrected age of 18 months; it was expressed as CAT-DQ, CLAMS-DQ and Full Scale (FS)-DQ. Statistical analysis using correlation test, with $\mathrm{p}<$ 0.05 being considered significant.

Results Individual expression of eHsc70 and eHsp70 was not correlates with either CAT-DQ, CLAMS-DQ or FS-DQ. However, simultaneously expression of $\mathrm{eHsc}+\mathrm{p} 70$ correlates significantly with CAT-DQ $(r=0.470 ; p=0.031)$, CLAMS-DQ $(r=$ $0.509 ; \mathrm{p}=0.018)$ and FS-DQ $(r=0.496 ; \mathrm{p}=0.022)$.

Conclusions This study supports the concept that circulating eHsc70 and eHsp70 at birth may have specific effects in brain development when they were expressed simultaneously. Possibly these proteins may function as a marker for cellular intervention in preterm-born children who show abnormal DQ.

\section{PS-253b GLUTEN FREE DIET FOR CHILDREN WITH ATTENTION DEFICIT AND HYPERACTIVITY DISORDER}

M Lykogeorgou, S Karkelis, O Papadaki-Papandreou, M Nikita. Paediatrics, Aghia Sophia Children's Hospital, Athens, Greece

\subsection{6/archdischild-2014-307384.554}

Background and aims Attention deficit and hyperactivity disorder (ADHD) is a complex neurodevelopmental-neurobiological disorder of behaviour, which is characterised by increased hyperactivity levels. Also, many of the children diagnosed with ADHD have significant nutritional problems. The aim of the study was to investigate the correlation of the hyperactivity status of these children with major nutritional changes using gluten free diet. 